\title{
Electron spin resonance study of puff-resolved free radical formation in mainstream cigarette smoke
}

\author{
Mariana Ghosh, ${ }^{a} *$ Chuan Liu, ${ }^{a}$ and Petre Ionita ${ }^{b}$ \\ ${ }^{a}$ GR\&D Centre, British American Tobacco, Regents Park Road, Southampton, SO15 8TL, U.K \\ ${ }^{b}$ Institute of Physical Chemistry, 202 Spl. Independentei, Bucharest 060021, Romania \\ E-mail: Mariana_Ghosh@bat.com
}

\begin{abstract}
Puff-by-puff resolved gas phase free radicals were measured in mainstream smoke from Kentucky 2R4F reference cigarettes using ESR spectroscopy. Three spin-trapping reagents were evaluated: PBN, DMPO and DEPMPO. Two procedures were used to collect gas phase smoke on a puff-resolved basis: $i)$ the accumulative mode, in which all the gas phase smoke up to a particular puff was bubbled into the trap (i.e., the $5^{\text {th }}$ puff corresponded to the total smoke from the $1^{\text {st }}$ to $5^{\text {th }}$ puffs). In this case, after a specified puff, an aliquot of the spin trap was taken and analysed; or, ii) the individual mode, in which the spin trap was analysed and then replaced after each puff. Spin concentrations were determined by double-integration of the first derivative of the ESR signal. This was compared with the integrals of known standards using the TEMPO free radical. The radicals trapped with $\mathrm{PBN}$ were mainly carbon-centred, whilst the oxygen-centred radicals were identified with DMPO and DEPMPO. With each spin trap, the puff-resolved radical concentrations showed a characteristic pattern as a function of the puff number. Based on the spin concentrations, the DMPO and DEPMPO spin traps showed better trapping efficiencies than PBN. The implication for gas phase free radical analysis is that a range of different spin traps should be used to probe complex free radical reactions in cigarette smoke.
\end{abstract}

Keywords: Cigarette smoke, spin trap, PBN, DMPO, DEPMPO, tempo

\section{Introduction}

Smoke formation inside a burning cigarette is governed by many complex and dynamic physical and chemical processes. Understanding these processes plays an important role in designing cigarettes with reduced toxicant levels. Broadly speaking, mainstream smoke emitted from the filter end of a cigarette following a puff is first generated by combustion and pyrolysis reactions in the burning zone (or coal). This is followed by a series of physical (e.g., filtration and condensation) and chemical interactions as the smoke is drawn down the cigarette rod. ${ }^{1}$ The tobacco rod itself is consumed and otherwise modified during this process and therefore each 
puff operates in a different environment. Analysis of the smoke generated on a puff by puff basis can often give valuable insights into the above mentioned mechanisms. For example, the mainstream nicotine-free-dry-particulate-matter (or 'tar') obtained by a smoking machine shows a gradual increase on a puff-by-puff basis. This is mainly caused by a combination of the reduced air dilution through cigarette paper and the re-pyrolysis of the particulate matter that is condensed on the rod from the previous puffs. ${ }^{1,2}$

Although the dynamic and reactive nature of cigarette smoke is recognized, it is only recently that studies based on fast mass spectrometers and laser-based techniques have begun to unravel the extent of formation of transient smoke species. ${ }^{3-14}$ Routine smoke analysis methods used in the tobacco industry are usually developed to quantify the total yields of smoke constituents after the entire cigarette is machine-smoked under a standard puffing regime. To provide the accuracy and sensitivity required for puff-resolved analysis, fast analytical techniques are needed. The information gathered in this way gives a more in-situ description of the smoke formation process, and is potentially more relevant to understanding how smokers are exposed to cigarette smoke. ${ }^{3-7}$ Thus, in addition to the gradual increase in the 'tar' yields with puff number, some other trends have also been observed. ${ }^{7-10}$ For example, it has been shown that, for some vapour phase smoke components (e.g., formaldehyde and 1,3-butadiene), the yields for the first puff are much higher than for the subsequent puffs and can account for over 30 to $40 \%$ of the total yields. ${ }^{11-14}$ This phenomenon may be related to the fact that the tobacco consumed in the $1^{\text {st }}$ puff is heated from room temperature, while for later puffs the tobacco is pre-heated by the advancing smouldering coal. ${ }^{3}$ Different lighting methods can also affect the yields in the first puff. ${ }^{3-13}$

It is well known that free radicals in cigarette smoke contain both short-lived gas phase radicals and persistent particulate phase radicals. ${ }^{15-17}$ Using electron spin resonance (ESR) spectroscopy, it is possible to quantify as well as identify the radical species involved. The shortlived free radicals, which are difficult to detect directly by ESR, are normally captured by a spintrap (a diamagnetic molecule), forming a persistent spin-adduct which is then analysed by ESR. $^{18,19}$

Previous work showed that carbon- and oxygen-centred radicals are mainly formed in the gas phase smoke, while the particulate phase contains semiquinone radicals. ${ }^{18-24}$ As far as the authors are aware, there have been no published studies of free radical behaviour in cigarette smoke on a puff-by-puff basis.

Different spin-traps have different selectivities towards radical capture. Hence a range of spintraps is required to study a complex system such as cigarette smoke. By applying different spintraps with different selectivities, it was hoped to distinguish a range of different free radicals in cigarette smoke. Three spin-traps were selected in this work, namely PBN ( $N$ - $t$-butyl- $\alpha$ phenylnitrone), DMPO (5,5-dimethyl-1-pyrroline- $N$-oxide), and DEPMPO (diethoxyphosphoryl5-methyl-1-pyrroline- $N$-oxide). Their structures are given in Figure 1. 

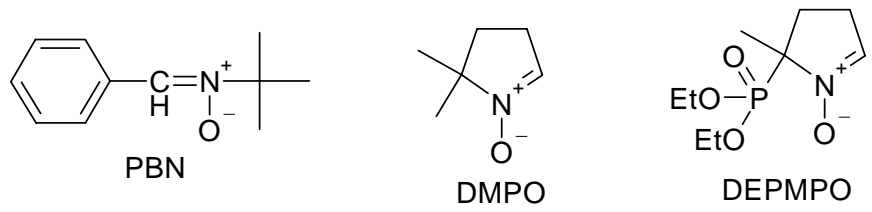

Figure 1. Structures of the three spin-traps employed in this study.

\section{Results and Discussion}

\section{PBN Experiments}

The spectra recorded with the PBN trap showed a triplet of double peaks with the hyperfine coupling constants at $\mathrm{a}_{\mathrm{N}}=1.42 \mathrm{mT}$ and $\mathrm{a}_{\mathrm{H}}=0.205 \mathrm{mT}$. Figure 2 shows typical spectra recorded in the individual procedure (Figure 2a) and in the accumulative procedure (Figure 2b); the experimental spectra (in red) are superposed on the simulated ones (in black). The results of the simulation (for details see Experimental part) are consistent with the formation of a carboncentred radical, which accounts for $c a$. 85-100 \% of the total radicals trapped by PBN for all the puffs. The spectrum recorded for the first puff showed that it consisted almost exclusively of carbon-centred radicals (as in Figure 2a), while for puff numbers 2 to 9, there were small amounts (up to $15 \%$ ) of the oxidation product from the starting PBN spin-trap (PBN-ox) with $\mathrm{a}_{\mathrm{N}}=0.84 \mathrm{mT}$ (as in Figure $2 \mathrm{~b}$ ). An un-identified free radical with a hyperfine coupling $\mathrm{a}_{\mathrm{N}}=0.76$ $\mathrm{mT}$ was also found (less than $10 \%$ ).

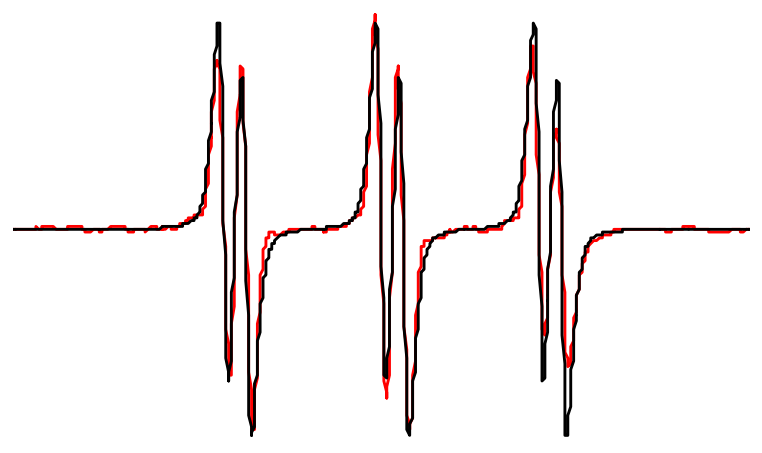

(a)

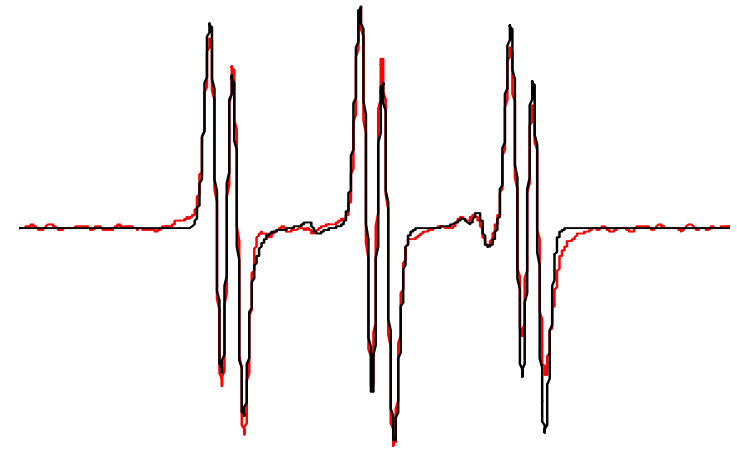

(b)

Figure 2. Typical ESR spectrum recorded for individual (a) and accumulative (b) experiments with PBN: small signals from PBN-ox can be noticed in the later case.

Using the individual procedure, each puff contained about $1.5-2 \times 10^{14}$ spins (Figure 3a). In the accumulative procedure (Figure $3 b$ ), the total concentration of the free radicals increased with puff number from about $2 \times 10^{14}$ spins after the first puff, to about $13 \times 10^{14}$ spins after the 
$8^{\text {th }}$ puff. The dashed line in Figure $3 \mathrm{~b}$ depicts the sum of the spin concentrations measured by the individual procedure (i.e., the sum of the data in Figure 3a). The close match between the two measurements apart from the $9^{\text {th }}$ puff shows that the PBN traps are stable and maintain their efficiencies over the approximately 10 min time period of the experiment. A clear reduction in free radical concentrations was observed in the $9^{\text {th }}$ puff ( $12 \times 10^{14}$ spins), and this could be due to the loss of trapping efficiency as a result of prolonged exposure of the spin trap to the gas phase free radicals. At this stage, there is no further experimental evidence to explain the loss of efficiency. This result demonstrated that the individual and accumulative procedures for the PBN traps have similar performances for trapping gas phase free radicals.

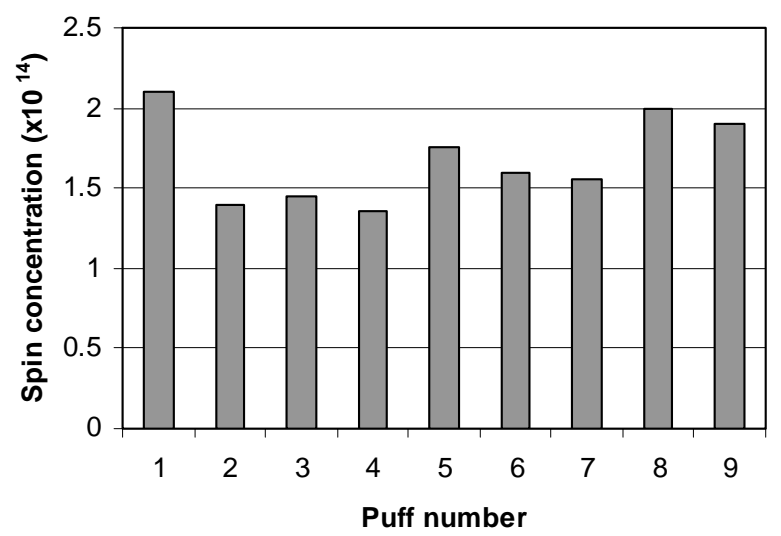

(a)

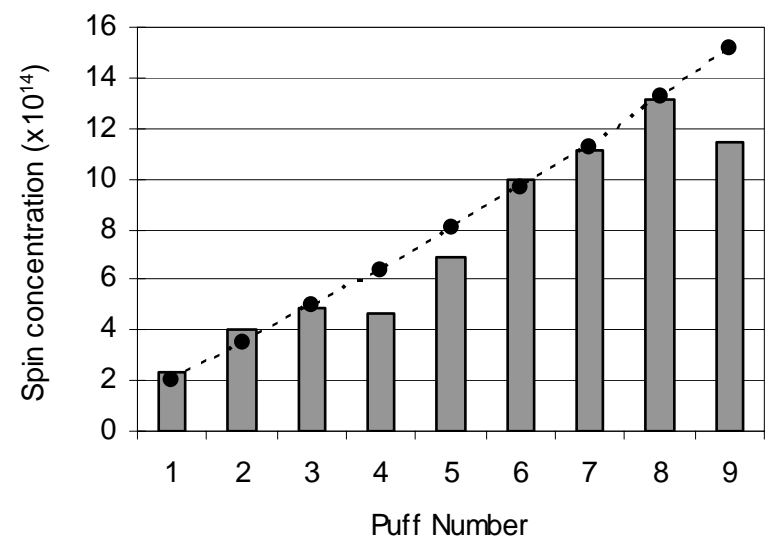

(b)

Figure 3. Variation of spin concentration with puff number in the individual (a) and accumulative (b) procedures for PBN.

\section{DMPO Experiments}

As shown in our previous work, ${ }^{18}$ the spectra recorded with DMPO in the final accumulative procedure (i.e., after all the 9 puffs were collected) revealed a set of hyperfine coupling constants predominantly associated with oxygen-centred radicals. Furthermore, the simulation that best matched the data was obtained by assuming a mixture of oxygen-centred and carbon-centred radicals together with some decomposition products. In this study, the spectrum from the $1^{\text {st }}$ puff (Figure 4a) was simulated with $c a$. 68\% oxygen-centred radicals comprising a mixture of two oxygen-centred spin adducts, the first one with $\mathrm{a}_{\mathrm{N}}=1.295 \mathrm{mT}, \mathrm{a}_{\mathrm{H} 1}=0.668 \mathrm{mT}, \mathrm{a}_{\mathrm{H} 2}=0.176 \mathrm{mT}$, and the second one with $\mathrm{a}_{\mathrm{N}}=1.32 \mathrm{mT}, \mathrm{a}_{\mathrm{H} 1}=0.75 \mathrm{mT}, \mathrm{a}_{\mathrm{H} 2}=0.18 \mathrm{mT}$. In addition, the simulation included ca. 9.9\% carbon-centred radicals ( at $\mathrm{a}_{\mathrm{N}}=1.437 \mathrm{mT}, \mathrm{a}_{\mathrm{H}}=2.165 \mathrm{mT}$ ) and $c a .22 \%$ of an unknown radical species $\left(a^{a} a_{N}=1.35 \mathrm{mT}\right.$ ). After the $9^{\text {th }}$ puff, there was a decrease in the level of the carbon-centred radicals together with an increase in the level of the decomposition products (Figure 4b).

For the free radicals trapped by the individual procedure, all the puffs contained a small amount of carbon-centred radicals. The overall spectra were still dominated by the two major 
oxygen-centred radicals. The intensity of the spectra (Figure 5a) was about 3-4 x $10^{14}$ spins/puff, which is approximately twice the concentration found in PBN (Figure 3a). This suggests that DMPO is a more efficient spin-trap than PBN for the oxygen-centred radicals when the individual procedure is used. Figure $5 \mathrm{~b}$ compares the spin concentrations obtained in the DMPO for both the sum of the individual procedures (the dashed line) and for the accumulative procedure (the bar chart). It is clear from the figure that the sums of the spin concentrations obtained from the individual puffs were much higher than those measured at each puff by the accumulative procedure, and this trend starts as early as the $2^{\text {nd }}$ puff. This indicates that the DMPO begins to lose its stability after approximately 2 minutes, making the accumulated procedure much less efficient in trapping the radicals.

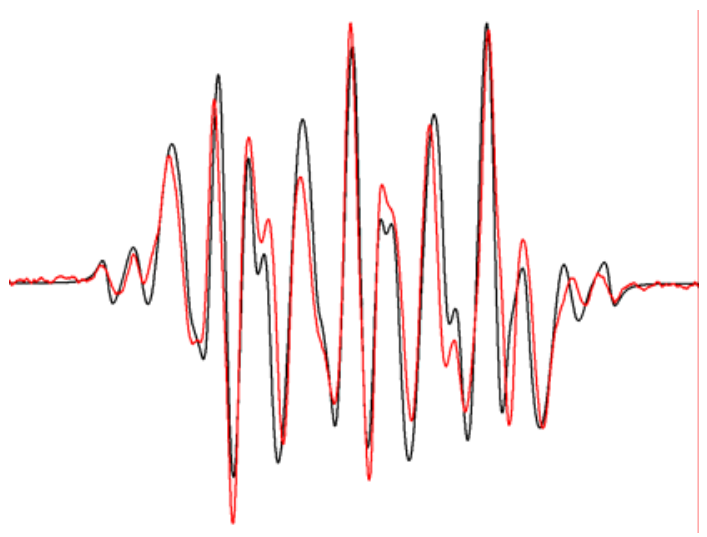

(a)

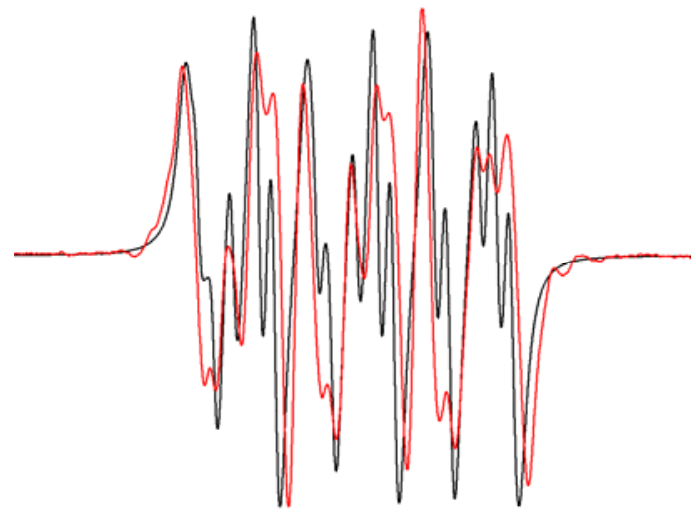

(b)

Figure 4. Experimental (red) and simulated ESR (black) spectrum after the $1^{\text {st }}(a)$ and the $9^{\text {th }}$ puffs $(b)$. The individual procedure with DMPO.

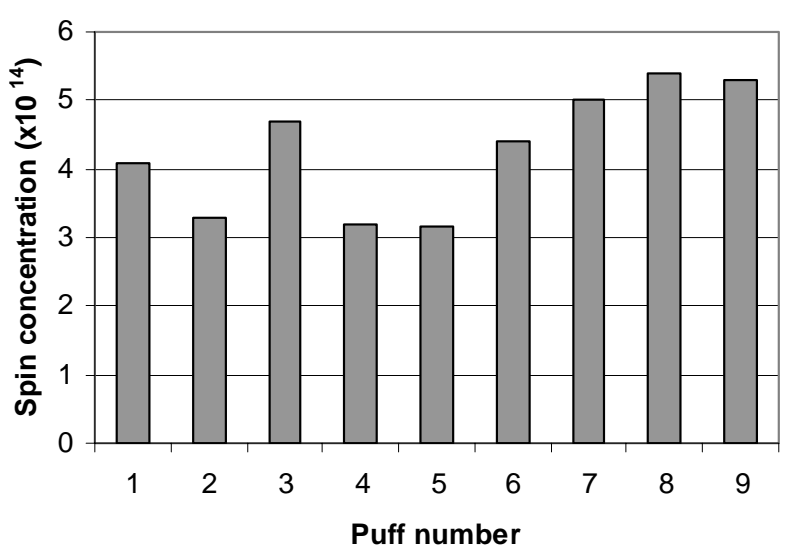

(a)

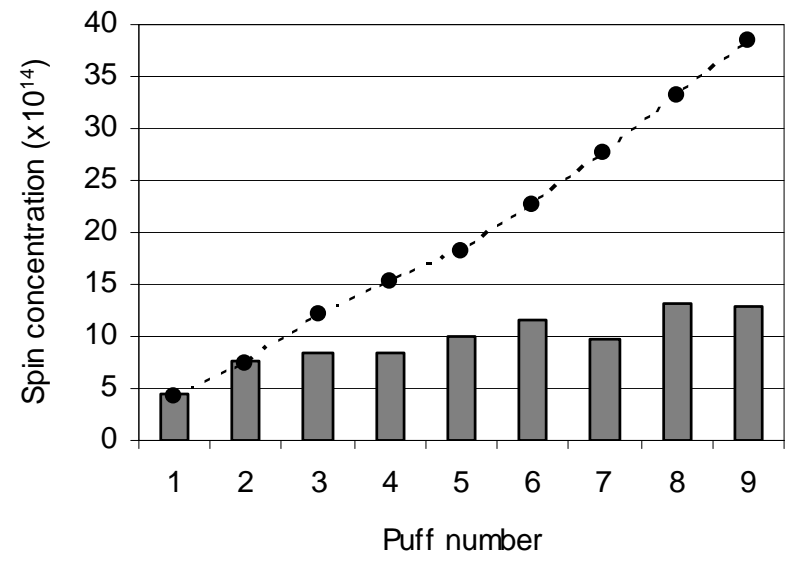

(b)

Figure 5. Variation of spin concentration with puff number in the individual (a) and accumulative procedure (b) for DMPO. 


\section{DEPMPO Experiments}

The spectra obtained with the DEPMPO spin trap are more complex (Figure 6), as for DMPO the spectra were dominated by oxygen-centred radicals. The features of the spectrum were very similar for each puff. The numbers of carbon-centred radicals decreased with puff number and the greatest numbers were seen in the first puff, as was found for DMPO. For the accumulative procedure the amount of radicals increased with puff number to ca. $30 \times 10^{14}$ spins, demonstrating that among the three spin traps investigated DEPMPO had the highest capacity to trap short-lived gas phase radicals. Despite the complexity, the spectra could be modelled (Figure 6) and the hyperfine coupling constants obtained were close to those reported in the literature (carbon-centred radicals: $\mathrm{a}_{\mathrm{N}}=1.372 \mathrm{mT}, \mathrm{a}_{\mathrm{H}}=2.319 \mathrm{mT}$, and $\mathrm{a}_{\mathrm{P}}=4.623 \mathrm{mT}$; for oxygen-centred radical: $\mathrm{a}_{\mathrm{N}}=1.382 \mathrm{mT}, \mathrm{a}_{\mathrm{H}}=0.82 \mathrm{mT}$, and $\mathrm{a}_{\mathrm{P}}=4.511 \mathrm{mT}$ ).

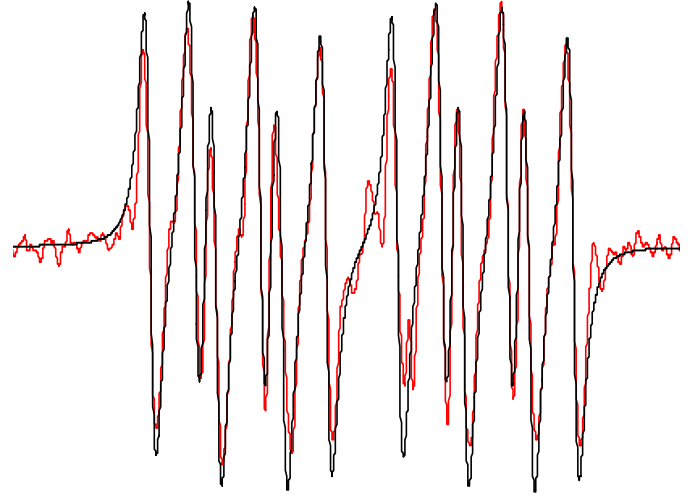

(a)

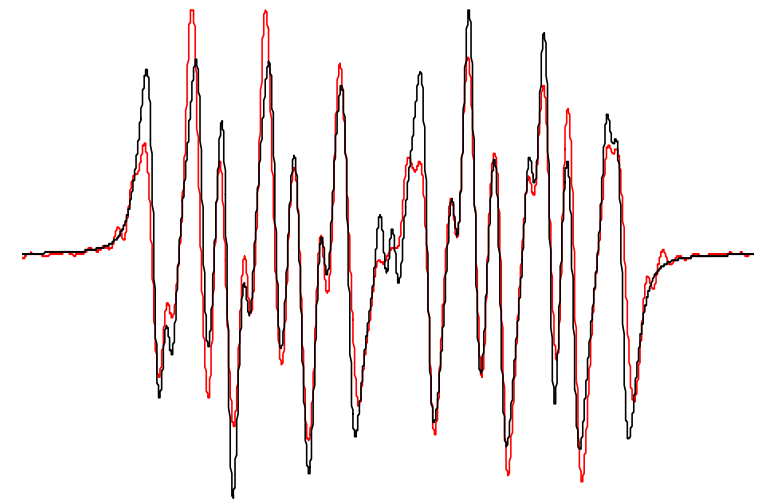

(b)

Figure 6. A comparison between experimental (red) and simulated ESR spectrum (black) for the last puff in the individual (a) and accumulative (b) procedure with DEPMPO.

Simulation with the hyperfine coupling constants $\mathrm{a}_{\mathrm{N}}=1.258 \mathrm{mT}, \mathrm{a}_{\mathrm{H}}=0.843 \mathrm{mT}$ and $\mathrm{a}_{\mathrm{P}}=4.721$ mT suggested an HO spin adduct of DEPMPO. This finding should be a genuine one, as the spectra were recorded in benzene, and not in water, where non-radical nucleophilic substitution may occurs. ${ }^{25}$ It is well known that this type of spin-adduct is easily obtained at high $\mathrm{pH}$ in the presence of water (via nucleophilic substitution), but it seems very unlikely that the water formed in the combustion process may reacts in the gas phase to form DEPMPO-OH spin adduct. A recent work showed that catalaze enzyme immobilized on the cigarette filter reduce the peroxides and other pollutants in cigarette smoke. ${ }^{26}$ The spin concentration per puff obtained using the individual procedure was around ca. 3-4 x $10^{14}$ spins/puff. This gives the DEPMPO and DPMO a similar trapping efficiency based on the individual procedure. However, Figure $7 \mathrm{~b}$ shows that the sum of the spin concentrations from the DEPMPO was slightly higher than those obtained by the accumulative procedure for the first four puffs and was essentially the same until the $8^{\text {th }}$ puff. In other words, the stability of the DEPMPO was much higher than the DPMO spin traps and lasted until at least the $8^{\text {th }}$ puff. 


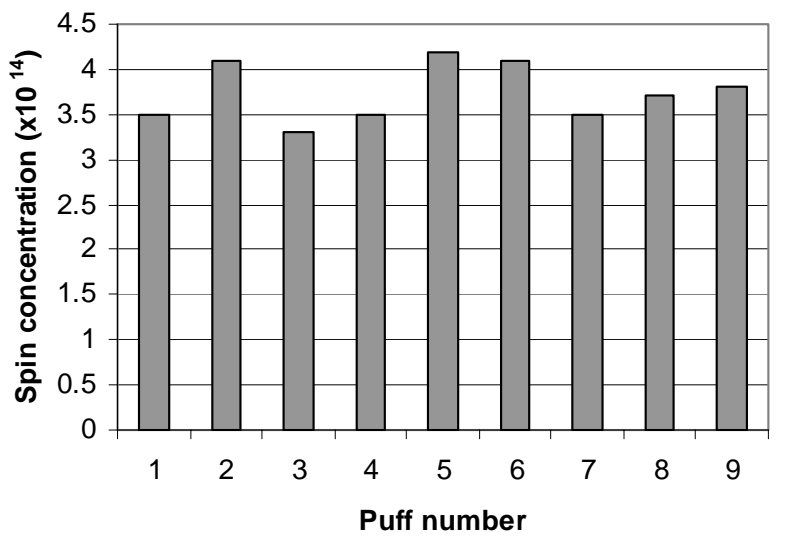

(a)

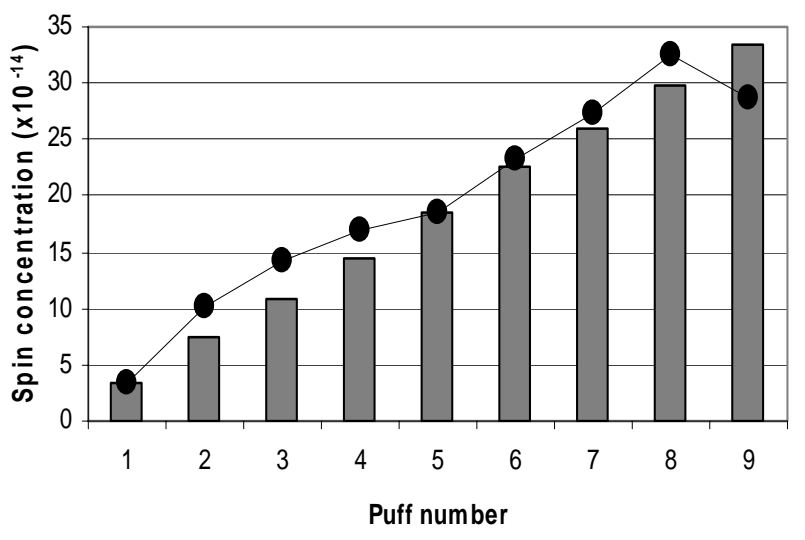

(b)

Figure 7. Variation of spin concentration with puff number in the individual (a) and accumulative procedure $(b)$ for DEPMPO.

Finally, Table 1 lists the hyperfine coupling constants and the types of radicals identified in this work.

Table 1. Hyperfine coupling constants (in mT) obtained for PBN, DMPO and DEPMPO

\begin{tabular}{ccccc}
\hline Spin Trap & $\mathrm{a}_{\mathrm{N}}$ & $\mathrm{a}_{\mathrm{H}}$ & $\mathrm{ax}_{\mathrm{X}}$ & Remarks \\
\hline \multirow{3}{*}{ PBN } & 1.42 & 0.205 & - & C-centred radical \\
& 0.84 & - & - & PBN-ox \\
& 0.76 & - & - & Decomposition product \\
\hline \multirow{3}{*}{ DMPO } & 1.295 & 0.668 & 0.176 & O-centred radical \\
& 1.32 & 0.75 & 0.18 & O-centred radical \\
& 1.437 & 2.165 & - & C-centred radical \\
& 1.35 & - & - & Decomposition product \\
\hline \multirow{3}{*}{ DEPMPO } & 1.372 & 2.319 & 4.623 & C-centred radical \\
& 1.382 & 0.82 & 4.511 & O-centred radical \\
& 1.258 & 0.843 & 4.721 & HO- spin-adduct \\
\hline
\end{tabular}

\section{Conclusions}

In summary the three spin traps showed different affinities toward different gas phase free radicals in the mainstream smoke from the Kentucky reference 2R4F cigarette. The combined spin trapping and simulation methodology was able to detect the presence of carbon-centred and oxygen-centred free radicals on a puff-by-puff basis. The use of the individual and the accumulative procedures demonstrated the different stabilities of the three spin traps. Whilst this makes direct comparison of the information more difficult, it illustrates the need to select several 
traps with different characteristics when studying a complex system such as cigarette smoke. The other conclusions of this study are:

- PBN mainly trapped carbon-centred radicals and its efficiency for carbon-centred radicals was higher than that of either DMPO and DEPMPO;

- DMPO mainly detected oxygen-centred radicals and its stability deteriorated after approximately two minutes. The number of spins trapped by DMPO was almost twice that trapped by PBN;

- In DEPMPO, HO radicals were detected in addition to oxygen- and carbon-centred radicals. DEPMPO has a greater stability than DMPO and a similar stability to PBN;

- On the whole, the puff to puff variations in spin concentration were relatively small compared to some other gas phase smoke analytes. ${ }^{11-14}$

\section{Experimental Section}

General Procedures. All the ESR spectra were recorded at ambient temperature on a JEOL FR30EX spectrometer. The spectrometer had the following general settings: centre field $336 \mathrm{mT}$, sweep field $10 \mathrm{mT}$, frequency $9.42 \mathrm{GHz}$, power $2 \mathrm{~mW}$, sweep time $60 \mathrm{~s}$, time constant $0.1 \mathrm{~s}$, modulation frequency $100 \mathrm{kHz}$, gain 200 and modulation width $0.1 \mathrm{mT}$ (lower modulation width did not improve in any way the recorded spectra).

Cigarette smoking was carried out on a 20-port RM20 rotary smoking machine (Borgwaldt KC) operated under the ISO puffing parameters (35 ml puffs, 2 seconds duration, one puff every 60 seconds). A 3-necked, pear-shaped $25 \mathrm{ml}$ flask, which held the spin trapping solution, was placed downstream after a Cambridge filter holder which separated the gas and particulate phases of the smoke. The flask was connected to the Cambridge filter holder with a $5 \mathrm{~cm}$ long PTFE tube.

Kentucky 2R4F reference cigarettes (University of Kentucky, Kentucky Tobacco Research and Development Centre) were used in this work. Before smoking, the cigarettes were conditioned for at least $48 \mathrm{hr}$ at $60 \%$ relative humidity and $20^{\circ} \mathrm{C}$. Nine cigarettes were smoked simultaneously.

Two methods were used to obtain gas phase smoke for analysis of individual puffs. The accumulative method: nine cigarettes were smoked and the spin-trap solution was not changed after a puff but an aliquot of the spin-trap was taken immediately after the puff for ESR analysis. The radicals detected therefore reflected the combined gas phase smoke up to this puff. The individual method: in this case, the spin-trap solution was changed after each puff with a fresh one. In this way only the gas phase radicals formed during the individual puff were analyzed.

A $0.01 \mathrm{M}$ solution of the spin-trapping agent in $5 \mathrm{ml}$ benzene was placed in the pear-shaped flask. In the case of the accumulative procedure, an aliquot of $0.4 \mathrm{ml}$ of the trapping solution was used for ESR analysis and the same amount of the clean trapping solution was added back to maintain the $5 \mathrm{ml}$ total volume. The sample was deoxygenated using a (liquid nitrogen) freeze- 
pump-thaw technique at a pressure of $10^{-3} \mathrm{~mm} \mathrm{Hg}$. This step was necessary to obtain clearly resolved ESR spectra, as the presence of atmospheric oxygen dramatically reduces the signal intensity and broadens the ESR peaks. ${ }^{18,19}$

The concentration of the free radicals was estimated by a double-integration procedure, i.e., the double integral of an experimental ESR spectrum was compared to that of a standard stable radical (TEMPO) of a known concentration. The ratios between different types of radicals were determined by simulating the ESR spectra [18]. Briefly, the experimental spectra were first digitalized by a commercial software (JEOL), and then simulated spectra were generated using WinSim software (version 2002 from: http://epr.niehs.nih.gov/pest.html).

\section{Acknowledgements}

P. I. thanks GR\&D (British American Tobacco) for the opportunity to work as a visiting scientist.

\section{References}

1. Baker, R. R. Smoke Chemistry, In Tobacco production, chemistry and technology: David, D. L.; Nielsen, M. T., Eds. Blackwell Science: Oxford, UK, 1999, p 398.

2. Norman, A. Cigarette Design and Materials, In Tobacco production, chemistry and technology: David, D. L.; Nielsen, M. T., Eds. Blackwell Science: Oxford, UK, 1999, p 353.

3. Parrish, M. E.; Harward, C. N. Appl. Spectrosc. 2000, 54, 1665.

4. Wagner, K. A.; Higby, R.; Stutt, K. Beitrag. Tabakforsch. Int. 2005, 21, 272.

5. Thomas, C. E.; Koller, K. B. Beitr. Tabakforsch. Int. 2001, 19, 345.

6. Parrish, M. E.; Lyons-Hart J. L.; Shafter, K. H. Vib. Spectrosc. 2001, 27, 29.

7. Li, S.; Banyasz, J. L.; Parrish, M. E.; Lyons-Hart, J.; Shafter, K. H. J. Anal. Appl. Pyrol. 2002, 65, 137.

8. Shi, Q.; Nelson, D. D.; McManus, J. B.; Zahniser, M. S.; Parrish, M. E.; Baren, R. E.; Shafter K. H.; Harward, C. N. Anal. Chem. 2003, 75, 5180.

9. Baren, R. E.; Parrish, M. E.; Shafer, K. H.; Harward, C. N.; Shi, Q.; Nelson, D. D.; McManus, J. B.; Zahniser, M. S. Spectrochim. Acta (A) 2004, 60, 3437.

10. Adam, T.; Ferge,T.; Mitschke, S.; Streibel, T.; Baker, R. R.; Zimmermann, R. Anal. Bioanal. Chem. 2005, 381, 487.

11. Mitschke, S.; Adam, T.; Streibel, T.; Baker, R. R.; Zimmermann, R. Anal. Chem. 2005, 77, 2288.

12. Adam, T.; Mitschke, S.; Streibel, T.; Baker, R. R.; Zimmermann, R. Anal. Chim. Acta 2006, 572, 219. 
13. Adam, T.; Mitschke, S.; Streibel, T.; Baker, R. R.; Zimmermann, R. Chem. Res. Toxicol. 2006, 19, 511.

14. Adam, T.; Baker, R. R.; Zimmermann, R. Anal. Bioanal. Chem. 2007, 387, 575.

15. Lyons, M. J.; Gibson, J. K.; Ingram, D. J. E. Nature 1958, 181, 1003.

16. Zhou, J.; Wu, K.; Sun, Y.; Cong, J. B.; Wang, C. Z.; Chang, X.; Xian, H.; Zhu, Y. F. Acta Chim. Sinica 2008, 66, 216.

17. Zhou, J.; Wu, K.; Sun, Y.; Cong, J. B.; Wang, C. Z.; Chang, X.; Xian, H.; Zhu, Y. F. Chem. J. Chinese U. 2007, 28, 1846.

18. Ghosh, M.; Ionita, P.; McAughey, J.; Cunningham, F. Arkivoc 2008, (xii), 74.

19. Baum, S. L.; Anderson, I. G. M.; Baker, R. R.; Murphy, D. M.; Rowlands, C. C. Anal. Chim. Acta 2003, 481, 1.

20. Nishizawa, M.; Kohno, M.; Nishimura, M.; Kitagawa, A.; Niwano, Y. Chem. Pharm. Bull. 2005, 53, 796.

21. Valavanidis, A.; Haralambous, E. Redox Rep. 2001, 161.

22. Pryor, W. A.; Prier, D. G.; Church, D. F. Environ. Health Perspect. 1983, 47, 345.

23. Church, D. F.; Pryor, W. A. Environ. Health Perspect. 1985, 64, 111.

24. Culcasi, M.; Muller, A.; Mercier, A.; Clement, J. A.; Payet, O.; Rockenbauer, A.; Marchand, V.; Pietri, S. Chem-Biol. Interact. 2006, 164, 215.

25. Clement, J. L.; Gilbert, B. C.; Ho, W. F.; Jackson, N. D.; Newton, M. S.; Silvester, S.; Timmins, G. S.; Tordo, P.; Whitwood, A. C. Perkin Trans. 2 1998, 1715.

26. Lu, X.; Hua, Z.; Du, G.; Ma, X.; Cao, J.; Yang, Z.; Chen, J. Free Radic. Res. 2008, 42, 244. 